entry into the cord. We have not clarified the best, safest technique.

\section{Sedation}

An alert patient is potentially able to provide a warning if the procedure is going awry. Some centers make it a practice to deeply sedate all patients. The risks of this practice need to be evaluated to determine if patient safety is being compromised for patient comfort.

In conclusion, we are dedicated to the safest possible techniques to provide quality care to our patients. The incidence of complications from interventional techniques is so low that we are currently unable to measure the incidence. Medication management does not effectively solve these problems. Therefore, it behooves us to consider available evidence and look for the best possible therapeutic options for our patients. This communication suggests specific areas which we should further investigate.

\section{REERENCES}

1. Manchikanti L, Singh V. Interventional Pain Management: Evolving Issues for 2003. Pain Physician 2003; 6:125-137.

2. Nelson JW. Letter to the Editor. In response to Houten JK, Errico TJ. Paraplegia after lumbosacral nerve block. Spine J 2003; 2:88-89.

3. Baker R, Dreyfus P, Mercer S et al. Cervical transforaminal injection of corticosteroids into a radicular artery: A possible mechanism for spinal cord injury. Pain 2003; 103:211-215.

4. Houten JK, Errico TJ. Paraplegia after lumbosacral nerve root block: Report of three cases. Spine / 2002; 2:70-75.

5. Brouwers PJ, Kottink EJ, Simon MA et al. A cervical anterior spinal artery syndrome after diagnostic blockade of the right C6nerve root. Pain 2001; 91:397-399.

6. Berger JJ, Hawkins IF. Celiac plexus injection: Use of a blunt-tipped needle. Regional Anesthesia 1995; 20 (2S).

7. Akins EW, Hawkins IF Jr., Mladinich C et al. The blunt needle: A new percutaneous access device. AJR Am J Roentgenol 1989; 152:181-182.

8. Heavner JE, Racz GB, Jenigiri B et al. Shgarp vs blunt nedle: A comparative study of penetration of internal structures and bleeding in dogs. Pain Practice 2003. In Press

9. Aprill C, Rogers K. Cervical transforaminal injection of corticosteroids. ISIS Scientific Newsletter 2002; 4:21-32.

\section{Standiford Helm II, MD}

Medical Director

Pacific Coast Pain Management Center P.O. Box 2549

Lake Forest, CA 92690-0549

E-mail: drhelm@pcpmc.com

Joseph F. Jasper, MD

Medical Director

Advanced Pain Medicine Physicians

1628 South Mildred Street, Suite \#105

Tacoma, WA 98465-1613

E-mail: apmedicine@qwest.net

Gabor B. Racz, MD

Grover Murray Professor, Professor and Chair Emeritus Director Pain Services, Texas Tech University Health Sciences Center Lubbock, TX 79430-0001, E-mail paula.brashear@ttuhsc.edu

\title{
RISK OF TRANSFORAMINAL EPIDURAL INJECTIONS
}

To the Editor:

There have been recent reports of serious complications arising from the injection of steroids into the spinal neural foramina especially in the cervical region (1-5). These complications have followed the relatively routine interventional pain clinic procedure of therapeutic injection to treat radicular pain. The procedures typically involve fluoroscopically-guided needle placement into the neural foramen with injection of water soluble contrast to document needle position. Disastrous events including paraplegia, quadriplegia and death have occurred shortly after the subsequent injection of particulate steroid through the needle. At least some of these procedures have been performed by experienced practitioners presumably using well-accepted injection technique. As interventional pain specialists, it behooves us to objectively re-examine the risks and benefits of neural foraminal injection so that we may maintain safe and efficacious medical practice for the benefit of our patients with chronic spinal pain.
Historically, transforaminal steroid injections have been used increasingly over the past decade to diagnose and treat neck and upper extremity pain syndromes. From review and extrapolation of insurance data, it is estimated that some 100,000 cervical transforaminal steroid injections have been performed in the United States in the past 10 years.

Review of the complications has revealed the following commonalities:

1. A sharp-tipped needle was placed into the neuroforamen under fluoroscopic guidance.

2. Contrast was injected and apparently documented good needle position.

3. A particulate steroid was injected subsequent to injection of contrast.

4. The complication occurred within minutes of the steroid injection.

5. Patient evaluation after the event revealed extensive spinal cord infarction as the pathological event. Most interventional pain specialists agree that the transforaminal approach to the epidural space has advantages in the diagnosis and treatment of certain pain syndromes (6-24). When irritation or inflammation of a specific spinal nerve root or dorsal root ganglion is suspected as the cause of intractable pain, transforaminal injection is the most direct route to apply medication to the pain generator. Furthermore, transforaminal injections are more likely to distribute therapeutic steroid to the anterior epidural space, which is the region most likely affected by irritation from bulging or herniated intervertebral discs.

\section{Spinal Cord Blood Supply}

Certainly, injection into the foramen poses unique risks because of the presence of foraminal arteries that are not present in the posterior epidural space. The spinal cord receives a rather tenuous blood supply through an anastomotic arterial network of feeder vessels. Many of these vessels are end arteries and collateral blood supply is lacking throughout much of the 
cord. The spinal arterial network is in turn fed by radicular arteries that course with the nerve root through various neural foramina en route to the cord. These radicular arteries are variable in number and location. In the thoracolumbar region the artery of Adamkiewicz exits the aorta and usually enters the spinal canal through a lower thoracic or upper lumbar foramen most commonly from the left side (2). An ascending sacral radicular artery and a second thoracic radicular artery may also contribute to thoracolumbar cord blood supply. In the cervical region, the vertebral arteries give rise to the anterior spinal artery at the upper cervical cord. The anterior spinal artery then descends through the spinal canal supplying the upper cord. The anterior spinal artery receives contributions from one or more radicular arteries, which originate from the more proximal vertebral artery and course through the cervical neural foramen with the nerve root. The most common scenario involves a single radicular feeder artery exiting the right proximal vertebral artery and following the $\mathrm{C} 6$ nerve root into the spinal canal through the right C5-6 neural foramen.

\section{Minimizing Risk}

In order for the complication of cord infarction to result from injection into the neural foramen, two conditions must be:

1. The tip of the needle must lie within an artery that is supplying the arterial feeder system of the cord.

2. Either placement of the needle itself or injection of material through the needle must cause downstream interruption of blood flow.

Arterial spasm from needle penetration is an unlikely cause of complication. The long and relatively safe history of angiography has demonstrated that needles can safely be passed into arteries without causing spasm. Embolization of the downstream spinal cord arteriolar system is a more likely explanation based on the distribution of infarction (anterior cord, Brown-Sequard lesion) associated with these events. Particulate steroid is like- ly the offending agent. Those of us who routinely use particulate steroids such as methylprednisolone and triamcinolone in pain clinic practice know that these compounds are capable of clogging the lumen of a 30 gauge needle. They are also capable of occluding blood flow as they are distributed to the arborized arteriolar network within the substance of the spinal cord. The fact that relatively large sections of the spinal cord even including portions of the brainstem have been infracted by single transforaminal injections attests to the fact that the radicular arteries arborize into a vast interconnected network of end-artery vessels.

Yet it seems clear that if we do not inject particulate steroid into the radicular artery, transforaminal injection is a relatively safe procedure. It is quite possible that the disastrous complications described above occurred either because the contrast flow pattern was intravascular but was incorrectly interpreted or because the needle tip migrated into the artery after the contrast was injected. No other explanation seems feasible. Meticulous injection technique will minimize the risk of intravascular injection and the following points should be considered:

1. Correct interpretation of the results of contrast injection is crucial to make absolutely certain that the nerve root and/or epidural space is outlined with contrast prior to injecting steroid. If contrast flows out of the needle tip and outlines the exiting nerve root, then by definition the needle tip cannot be intravascular.

a. Contrast should outline the exiting nerve root for a clear and definable distance laterally.

b. It is not necessarily a problem if contrast moves retrograde through the neuroforamen and into the epidural space but if the nerve root is not simultaneously visualized then it is difficult to determine whether the contrast moving medial is intravascular or epidural.

2. Once contrast injection has confirmed safe needle position, great pains must be taken to keep the needle completely stationary for the remainder of the procedure.

a. Use of a pigtail extension attached to the needle hub will facilitate exchange of contrast and medication syringes without moving the needle itself.

b. Injection of the active medication should be done incrementally with frequent fluoroscopic visualization to make sure the needle remains in proper position.

i. Subtle movement of the perineural contrast pool visualized during incremental injection of steroid indicates that the needle tip is remaining extra vascular.

In addition, some experts have recommended using blunt tip needles for transforaminal injection although these needles are not currently available in 25 gauge diameter making them less desirable for some practitioners. Blunt tip needles have been demonstrated to be less likely to penetrate vascular structures. Clear steroid preparations such has betamethasone may also decrease risk although it is unclear whether non-particulate steroid preparations will prove as efficacious as the particulate medications.

As evidenced by past experience and the long history of successful transforaminal injection, it is certainly possible to perform this procedure safely and with good result. Nonetheless, recent events demonstrate the potential for disaster with injection into the neuroforamen and each interventional pain specialist should reexamine the indications and techniques for this procedure. In addition, informed consent for transforaminal injection should include an explanation of the risk of spinal cord injury.

\section{David M. Schultz, MD}

Medical Advanced Pain Specialists 2104 Northdale Blvd NW, Suite 220

Minneapolis MN 55433

E-mail: dschultz@painphysicians.com 